\title{
Dynamic behavior of hot-wire probes in turbulent boundary layers
}

\author{
By J.M. Österlund \& A.V. Johansson \\ Department of Mechanics, Royal Institute of Technology 10044 Stockholm, Sweden
}

Published in Advances in turbulence V, 398-402, 1995

\begin{abstract}
Hot-wire measurements in air of $\overline{u w}^{+}$in the near wall region of a turbulent boundary layer at high Reynolds numbers indicate a thermal interaction for typical two-wire probes, such as X- and V-probes. In order to determine this interaction two infinitely long parallel wires have been studied numerically by solving the heat transfer equation. Computations give that for X- and V-probes a typical Péclet number based on wire separation should be larger than about 50 to keep thermal interaction at an acceptable level. On the other hand for the Parallel-probe the results indicate that the Péclet number should suitably be less than 10 to ensure good sensitivity and uniform response in the frequency range of interest.
\end{abstract}

\section{Experiments}

Experiments in a high Reynolds number turbulent boundary layer were carried out in the MTL-tunnel at KTH. The test section of the MTL-tunnel measures $1.2 \mathrm{~m} \times 0.8 \mathrm{~m} \times 7 \mathrm{~m}$. A $7 \mathrm{~m}$ long boundary layer plate has been constructed. It spans the entire width of the test section $(1.2 \mathrm{~m})$, has an elliptical leading edge and an adjustable flap. The use of miniaturized hot-wires is necessary for sufficient spatial resolution. We have developed the technique to build extremely small probes with the aid of a high accuracy micro-manipulator. The smallest probes built have wires of $0.6 \mu \mathrm{m}$ diameter and about $0.1 \mathrm{~mm}$ length. A new X-wire probe where the wires are contained within a box of $100 \mu \mathrm{m}$ side-length has also been built. A new type of probe where two wires are placed extremely close to each other have also been built (see fig. 1). The intention is to measure effects of varying temperature wake interaction from the two wires with varying flow angle. We have built such probes with a wire separation of about $10 \mu \mathrm{m}$. The aim is to replace the use of the necessarily rather large X-probes for near-wall measurements of the wall-normal velocity component. 


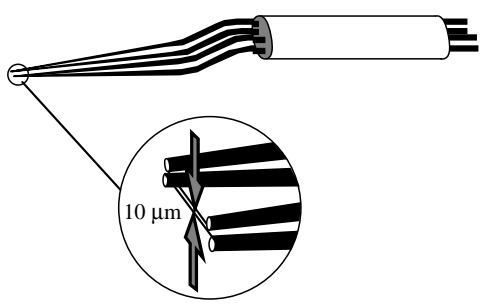

FiguRE 1. Parallel-probe.

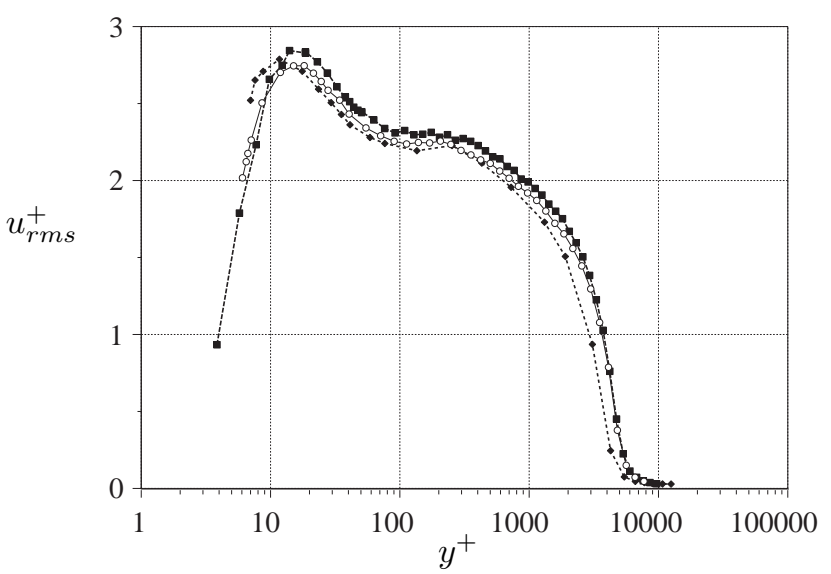

FIGURE 2. $u_{r m s}^{+}$profiles from single-probe, parallel-probe and x-probe. ०: X-wire probe with box size $175 \mu \mathrm{m}, R e_{\theta}=14500$. $\diamond$ : Parallel wire probe, $25 \mu \mathrm{m}$ division, $R e_{\theta}=7000$. $\square$ : Singlewire, $R e_{\theta}=14500$.

\section{Experimental results}

A large number of measurement series with a variety of hot-wire probes have been carried out in the MTL tunnel for Reynolds numbers up to $R e_{\theta}=14500$. Near-wall measurements in the upper part of this range are unique to the present experiments and constitute a fundamentally very important area of basic turbulence knowledge that so far is lacking. The experiments carried out so far have been successful (using very small probes) in terms of measuring 


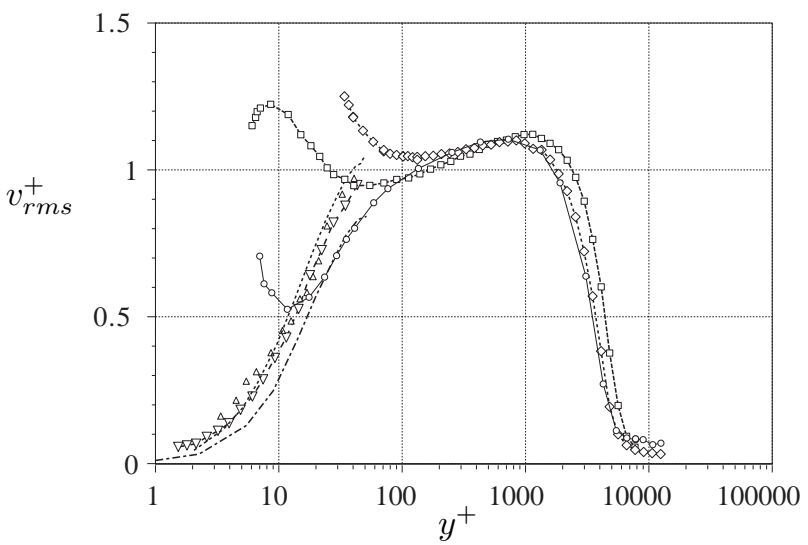

FiguRE 3. $v_{r m s}^{+}$profiles from parallel-probe and x-probe compared with data from measurements and simulations of channel and boundary layer flow. ○: Parallel-wire probe $25 \mu \mathrm{m}$ division, $R e_{\theta}=7000 . \diamond$ : X-wire probe boxsize $550 \mu \mathrm{m}$, $R e_{\theta}=7000$. $\square$ : X-wire probe boxsize $175 \mu \mathrm{m}, R e_{\theta}=14500$. Dashed line: DNS by Spalart (1988), $R e_{\theta}=1410$. Dashdotted line: DNS for channel flow by Kim et al. (1987). $\triangle$ : Measurements in oil channel by Kreplin \& Eckelmann (1979). $\nabla$ : LDV measurements in boundary layer by Karlsson \& Johansson (1988).

mean velocity and $u_{r m s}$ profiles and have shown consistency between measurements with single wire, X-wire and the new parallel wire probes (see figure 2 ). The results for $v_{r m s}$ are shown in figure 3 where data from a parallel wire probe and X-probes are compared (in the near wall region) with data from channel flow and boundary layer simulations. It is seen that all probes exhibit problems in the near wall region although the parallel wire probe seems to give the best results. The latter results appear to be reasonably accurate down to $y^{+}=15$. The large errors in the X-probe data in the near-wall region, motivated us to analyze the response of multi-wire probes in detail. The errors seen in figure 3 arise from interaction of the thermal wakes of the wires and becomes more accentuated with decreasing size of the probes. Also, the error in the Reynolds stress becomes considerably worse for the parallel-wire probe. Corresponding problems were found in measurements with $\mathrm{V}$-probes (for measurement of $\mathrm{u}$ and w). The calibration diagram of a $\mathrm{V}$-probe superimposed with 400 data points (see fig 4) indicate an artificial correlation between $\mathrm{u}$ and $\mathrm{w}$. Also the wide spread of the data points signifies an effect of the thermal wake interactions rather than truly large flow angles. 


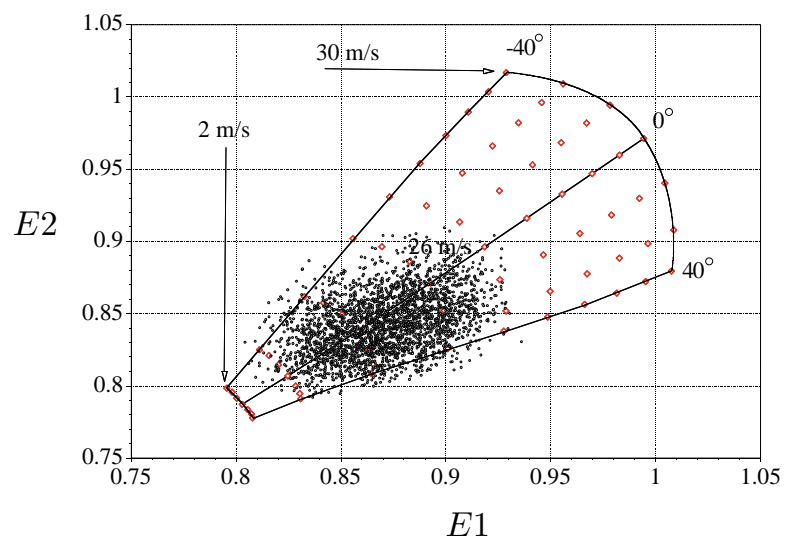

Figure 4. Calibration plot for the V-probe, superimposed with 4000 data samples taken at $y^{+}=6, U_{\infty}=27 \mathrm{~m} / \mathrm{s}, X=$ $5.5 \mathrm{~m}, \operatorname{Re}_{\theta}=14500$

\section{Numerical simulation of Parallel-probe}

An implicit algorithm for the two dimensional heat-transport equation has been implemented. An implicit algorithm would avoid most of the stability restrictions of an explicit scheme, but have the problem of achieving an efficient solution algorithm. The use of an operator splitting method (time-splitting) provides a way to get around this problem. By splitting each time-step into two half-steps where at each half step only terms associated with a particular coordinate direction are treated implicitly, only three implicit terms appear grouped on the main diagonal of the linear system. The efficient Thomas algorithm can be used to solve the system. Here a split formulation of the two-dimensional transport equation with a two-level Crank-Nicolson finite difference scheme is used on a rectangular grid. Constant temperature anemometry is simulated by forcing the temperature on the hot-wires to be constant. The fluctuating velocities in the turbulent boundary layer is simulated by a homogeneous velocity field, $\vec{v}=(U \cos \alpha, U \sin \alpha), \alpha=\alpha_{\max } \sin 2 \pi f t$. The heat flux from the wires is calculated at each time-step. The thermal interaction, if any, gives a difference in heat flux between the two wires.

\section{Results from numerical investigation of probe characteristics}

Computations were carried out for the case described above with two point sources of heat separated by a distance of $\delta$. This can either be regarded as an idealized model of a V-probe were the two wires have been idealized to point sources or as a more realistic model for a parallel-wire probe. A simple analysis 


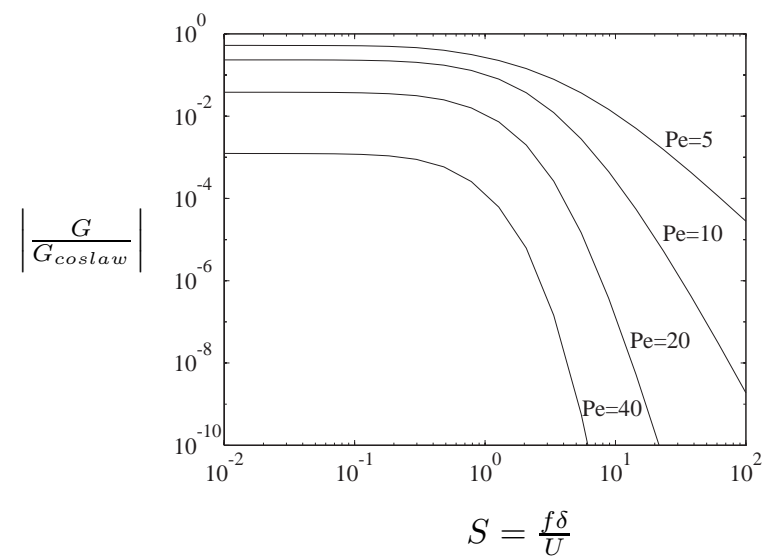

Figure 5. Amplitude of the transfer function $G$ for the response in $\mathrm{v}$ velocity normalized with the response of an ideal $\mathrm{X}$ - or V-probe.

of the situation yields that the results should best be described in terms of Nusselt numbers for the two wires as functions of two parameters, viz. the Péclet number of the probe $P e=\frac{U \delta}{\kappa}$ and the Strouhal number $S=\frac{f \delta}{U}$. The results of the computations are shown in figure 5 in terms of the maximum difference in Nusselt number between the two wires normalized with the corresponding difference for an ideal V-probe. This means that the curves can be interpreted as the sensitivity obtained with a parallel-wire probe normalized with that of an ideal V- (or X-) probe.

The phase shift of the parallel-probe (see fig 6) occurs at lower frequency than the decrease in magnitude. This indicates that correlations like $\overline{u v}$ may bee more sensitive to errors.

One may illustrate the above mentioned limitations by the situation in an airflow boundary layer at $y^{+}=3$ and a probe length $\mathrm{L}=0.2 \mathrm{~mm}$. For a free stream velocity of $25 \mathrm{~m} / \mathrm{s}$ the viscous length scale (at $\mathrm{x}=5 \mathrm{~m}$ ) is roughly 0.015 mm (giving $L^{+} \approx 13$ ). The Péclet number at this position becomes about 28 . A Strouhal number of 0.1 here corresponds to $1.5 \mathrm{kHz}$, which indicates that problems are likely to occur in this case.

\section{Conclusions}

For $\mathrm{X}$ - and V-probes the computations indicate that thermal interaction between the wire-wakes deteriorate the results for Péclet numbers below 50. For parallel-wire probes on the other hand the results suggest that the Péclet number should suitably be less than 10 . At a Pe of 5 the parallel wire has about $50 \%$ of the sensitivity of an $\mathrm{x}$ or v-probe. It is also clearly seen from figure 


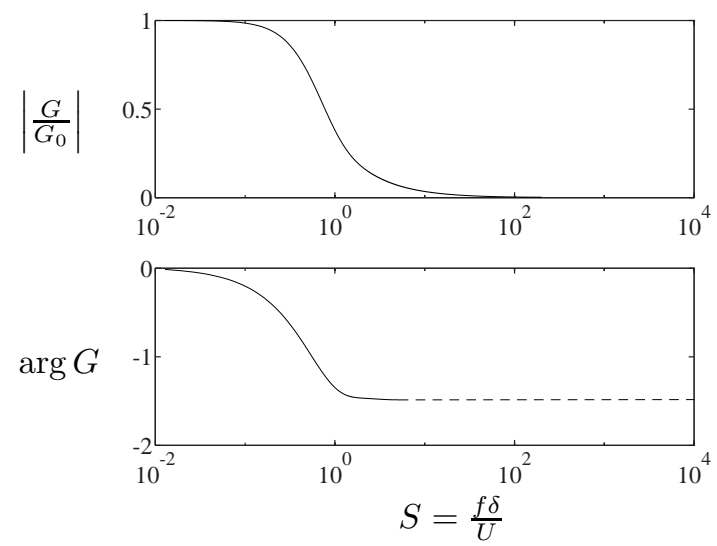

FiguRE 6. Frequency-response of parallel-probe normalized with response at zero frequency. $\mathrm{Pe}=10$.

5 that a static calibration only is valid up to Strouhal numbers of about 0.1 $\left(f<0.1 \frac{U}{\delta}\right)$.

Financial support from NUTEK is gratefully acknowledged. 


\section{References}

Gresko, JR., L. S. 1988 Characteristics of wall pressure and near-wall velocity in a flat plate boundary layer. Tech. Rep. 88-2. Fluid Dynamics Research Laboratory Report.

Karlsson, R. I. \& Johansson, T. G. 1988 LDV measurements of higher order moments of velocity fluctuations in a turbulent boundary layer. In Laser Anemometry in Fluid Mechanics. Ladoan-Instituto Superior Tećnico, 1096 L.C., Portugal.

Kim, J., Moin, P. \& Moser, R. 1987 Turbulence statistics in fully developed channel flow. J. Fluid Mech. 177, 133-166.

Kreplin, H.-P. \& Eckelmann, H. 1979 Behavior of the three fluctuating velocity components in the wall region of a turbulent channel flow. Phys. Fluids 22 (7).

SpalaRT, P. R. 1988 Direct simulation of a turbulent boundary layer up to $R e_{\theta}=$ 1410. J. Fluid Mech. 187, 61-98.

Willmarth, W. W. \& Bogar, T. J. 1977 Survey and new measurements of turbulent structure near the wall. Phys. Fluids 20 (10), 9-21. 